\title{
Gibberellin is not a regulator of miR156 in rice juvenile-adult phase change
}

\author{
Nobuhiro Tanaka
}

\begin{abstract}
Plant hormone gibberellin (GA) promotes juvenile-adult phase change in higher plants. To confirm the functions of GA in rice, I used dwarf mutant d18-dy. d18-dy is a loss-of-function allele of D18, which encodes GA30x2. d18-dy mutant exhibited long juvenile phase in morphological traits such as the size of the shoot apical meristem (SAM), shape of leaf blades, presence or absence of midribs and node-internode differentiation in stem. In contrast, expression patterns of juvenile-adult phase change markers miR156 and miR172 were similar between wild type and d18-dy. In addition, d18-dy mutation and GA did not affect expression levels of downstream genes of miR156. GA does not function upstream of miR156 in juvenile-adult phase change.
\end{abstract}

Keywords: Gibberellin, miR156, miR172, OsSPLS

\section{Background}

Juvenile and adult phases are distinguished by several morphological markers (Lawson and Poethig 1995; Telfer et al. 1997; Asai et al. 2002). Juvenile-adult phase change is regulated by miR156, miR172 and gibberellin (GA) in many higher plants (Lawson and poethig 1995; Telfer et al. 1997; Wu and Poethig 2006; Wang et al. 2011; Tanaka et al. 2011). However, the molecular mechanism involved in the juvenile-adult phase change is still unclear.

In Arabidopsis, juvenile leaves are round without abaxial trichomes, and adult leaves are long and serrated with abaxial trichomes (Telfer et al. 1997). In maize, leaves have epicuticular wax in juvenile phase, and adult leaves have no wax (Lawson and Poethig 1995). From these morphological markers, a lot of juvenile-adult phase change related mutants are reported in Arabidopsis and maize (Moose and Sisco 1996; Chuck et al. 2007; Schwarz et al. 2008; Smith et al. 2009). The observation of these mutants revealed that miR156 had significant roles in juvenile-adult phase change (Wu and Poethig 2006; Chuck et al. 2007; Wu et al. 2009). In the early vegetative stage, transcription level of miR156 exceeds that of miR172, whereas in later vegetative stage, the inverse pattern is seen (Wu and Poethig 2006; Chuck et al.

Correspondence: antanaka@mail.ecc.u-tokyo.ac.jp

Graduate School of Agricultural and Life Sciences, University of Tokyo, Tokyo 113-8657, Japan

\section{Springer}

(c) 2012 Tanaka; licensee Springer. This is an Open Access article distributed under the terms of the Creative Commons Attribution License (http://creativecommons.org/licenses/by/2.0), which permits unrestricted use, distribution, and reproduction in any medium, provided the original work is properly cited.
2007). In Arabidopsis, overexpression of miR156 causes a prolonged juvenile phase (Wu and Poethig 2006). In addition, miR156 overexpressed mutant, Corngrass1 (Cg1) shows long juvenile phase in maize (Chuck et al. 2007). The glossy15 (gl15) mutant shortens the juvenile phase in the maize epidermis (Moose and Sisco 1996); GL15 is an AP2-like gene that is the target of miR172 (Lauter et al. 2005). In addition, miR156 also controls juvenile-adult phase change in trees (Wang et al. 2011). miR156 inhibits juvenile-adult phase change via repression of SQUAMOSA PROMOTER BINDING PROTEINLIKE (SPL) family genes resulting in decrease of miR172 (Wu et al. 2009). Thus, miR156 and miR172 are key regulators in the juvenile-adult phase change.

Plant hormone Gibberellin (GA) is involved in the regulation of plant growth. GA is also known as adult phase promoter in Arabidopsis and maize (Lawson and Poethig 1995; Telfer et al. 1997; Telfer and Poethig 1998). GA deficient mutant, ga1-3 exhibits dwarfism and glabrous leaf without abaxial trichome in Arabidopsis (Telfer et al. 1997). In maize $d 1$ and $d 3$ mutants, the expression of leaf epidermal wax is prolonged and the expression of leaf epidermal hairs is delayed compared with wild type (Lawson and Poethig 1995).

There are a few reports that examine the relationship between miR156 and GA (Schwarz et al. 2008; Wang et al. 2009). Because the expression level of SPL9 was similar in wild type and ga1-3 mutant in Arabidopsis 
(Wang et al. 2009), miR156 and GA related genes may function independently. However, the relationship between miR156 and GA in juvenile-adult phase change is not confirmative.

In rice, a lot of GA-biosynthesis-deficient mutants are reported (Sasaki et al. 2002; Sakamoto et al. 2004). However no reports described how GA is related to juvenileadult phase change in rice except Tanaka et al. (2011). Almost all molecular genetic studies of juvenile-adult phase change have been confined to Arabidopsis and maize. However, considerable number of morphological and physiological traits known to differ between the juvenile and adult phases are reported in rice, including the size of the shoot apical meristem (SAM), size and shape of leaf blades, presence of midribs, vascular orientation in the stem, node-internode differentiation, and photosynthetic rate (Itoh et al. 2005), thus rice is useful plant to understand the juvenile-adult phase change.

In this report, I examined $d 18-d y$ mutant from the stand point of juvenile-adult phase change. Expression patterns of $m i R 156$ and $m i R 172$ in $d 18-d y$ demonstrate that GA regulates juvenile-adult phase change independently of miR156-related pathway.

\section{Results}

\section{Vegetative phenotypes of $d 18-d y$}

To confirm the function of GA as adult phase promoter, I examined the phenotypes of GA deficient mutant, $d 18-d y$. Rice D18 encodes GA3ox2, and the loss of function allele $d 18-d y$ causes severe dwarfism (Figure 1A). $d 18-d y$ has
9 bp deletion in the first exon of GA3ox2 gene (Sakamoto et al. 2004). GA 3-oxidase oxidizes $\mathrm{GA}_{20}$ and $\mathrm{GA}_{9}$ to synthesize active $\mathrm{GA}, \mathrm{GA}_{1}$ and $\mathrm{GA}_{4}$. The GA3ox deficient mutant is convenient to understand the function of GA in juvenile-adult phase change. First, I examined leaf shape that is defined as the ratio of leaf blade length to width. In wild type, the ratio drastically increased with elevation of leaf positions (Figure $1 \mathrm{~B}$ ). In $d 18-d y$, even the ratio of the 7 th leaf blade was below that of wild type 3rd leaf (Figure 1B). Second, I evaluated the presence of midrib in wild type and $d 18-d y$ leaf blades. The extent of midrib formation is a good marker for evaluating the juvenility in rice (Tanaka et al. 2011). I measured relative midrib length from the base of leaf blade. In $d 18-d y$ 3rd and 4th leaf blades, midrib covered approximately $20 \%$ and $40 \%$ of leaf blade, respectively (Figure $1 \mathrm{C}-\mathrm{E}$ ). These values were approximately half of those of wild type (Figure 1E). In both wild type and $d 18-d y$ 6th leaf, midrib covered more than $70 \%$ of leaf blade (Figure 1E). These results suggest that $d 18-d y$ shows prolonged juvenile phase, and it enters adult phase at around 6th leaf stage.

Next, I observed the changing pattern of SAM size during development. In $d 18-d y$, SAM remained smaller than that of wild type till the 4th-leaf stage (Figure 2A). Additionally, $d 18-d y$ showed different node-internode differentiation. Above the 5th leaf, the stem has obvious node in wild type (Figure $2 \mathrm{~B}$ ). By contrast, node differentiation was not detectable until the insertion of 6 th leaf in $d 18-d y$ (Figure 2C). These stem structures also indicate that $d 18-d y$ has long juvenile phase. From these
A

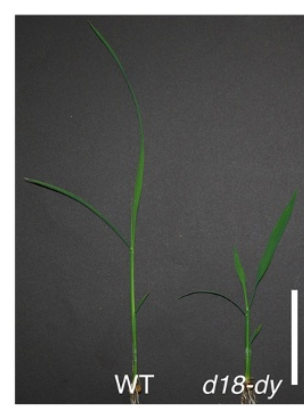

C

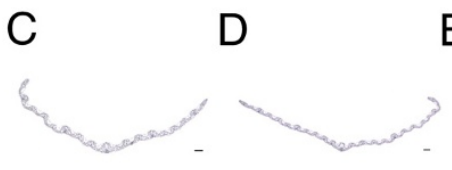

B

E
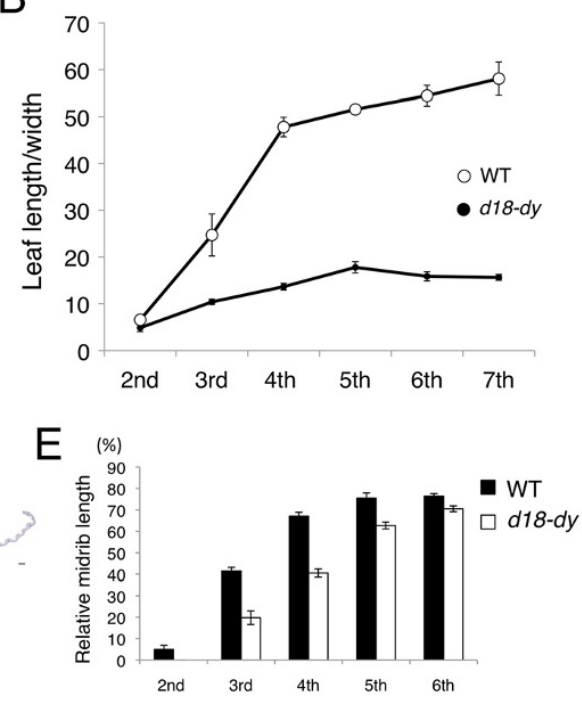

Figure 1 Phenotypes of leaf in $\mathbf{d} 1 \mathbf{8}$-dy plants. (A) 2-week-old wild type (Taichung 65 ) and $d 18-d y$. Bar $=5 \mathrm{~cm}$. (B) Change in the ratio of leaf blade length to width in wild type and d18-dy (C) Cross section of d18-dy 3rd leaf blade cut at $25 \%$ from the base. (D) Cross section of $d 18-d y 4$ th leaf blade cut at 45\% from the base. Bars $=100 \mu \mathrm{m}$. (E) Change of relative midrib length in leaf blades during development in wild type and d18-dy. Midrib length is shown as a percentage against total blade length. Data represent means \pm SD in $(\mathbf{B})$ and $(\mathbf{E})(n=5)$. 


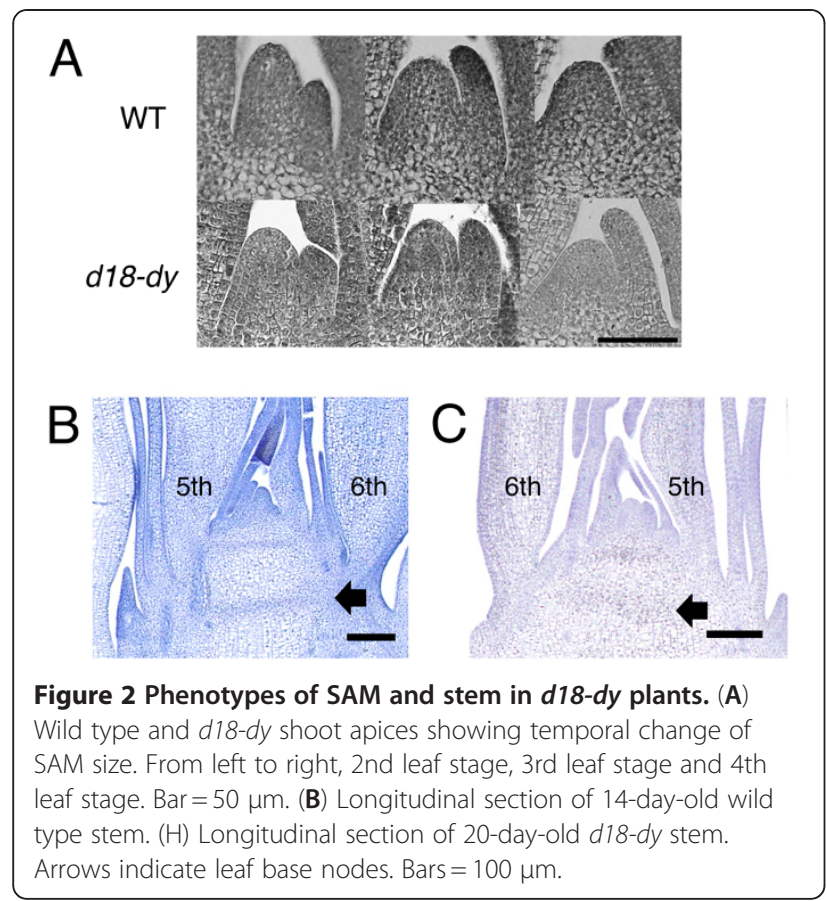

morphological traits in $d 18-d y$, I concluded that GA promoted juvenile-adult phase change in rice.

\section{GA and two miRNAs independently regulate juvenile-adult phase change}

To demonstrate the relationship between GA and two miRNAs in juvenile-adult phase change, I examined miR156 and miR172 expression patterns in wild type and $d 18-d y$ leaves (Figure 3A,B). In both wild type and $d 18-d y$ leaves, expression level of $m i R 156$ was the highest in 2nd leaf, rapidly decreased to approximately one-third in 3rd leaf (Figure 3A), and was maintained at low level until the 7th leaf (Figure 3A). Wild type and $d 18-d y$ also showed similar expression pattern of miR172: the expression was quite low in 2nd leaf, and increased dramatically toward the 7th leaf (Figure 3B). In conclusion, expression patterns of the two miRNAs in $d 18-d y$ were identical to those in wild type (Figure 3A,B). From normal expression patterns of two miRNAs and retarded juvenile-adult phase change in $d 18-d y$, I estimated that GA promotes adult phase transition independently of miR156 and miR172. To confirm the relationship between GA and two miRNAs, I treated wild type plants with $\mathrm{GA}_{3}$. There is a report that the expression level of GA deactivation gene, GA2ox4 is upregulated by GA treatment (Yamaguchi 2008). In GA treated plants, expression level of GA2ox4 was higher than in control plants (Additional file 1: Figure S1). This indicates that the experimental system of GA treatment is normally functioning. Subsequently I examined $m i R 156$ and $m i R 172$ expression levels in control and $\mathrm{GA}_{3}$ treated plants. Both
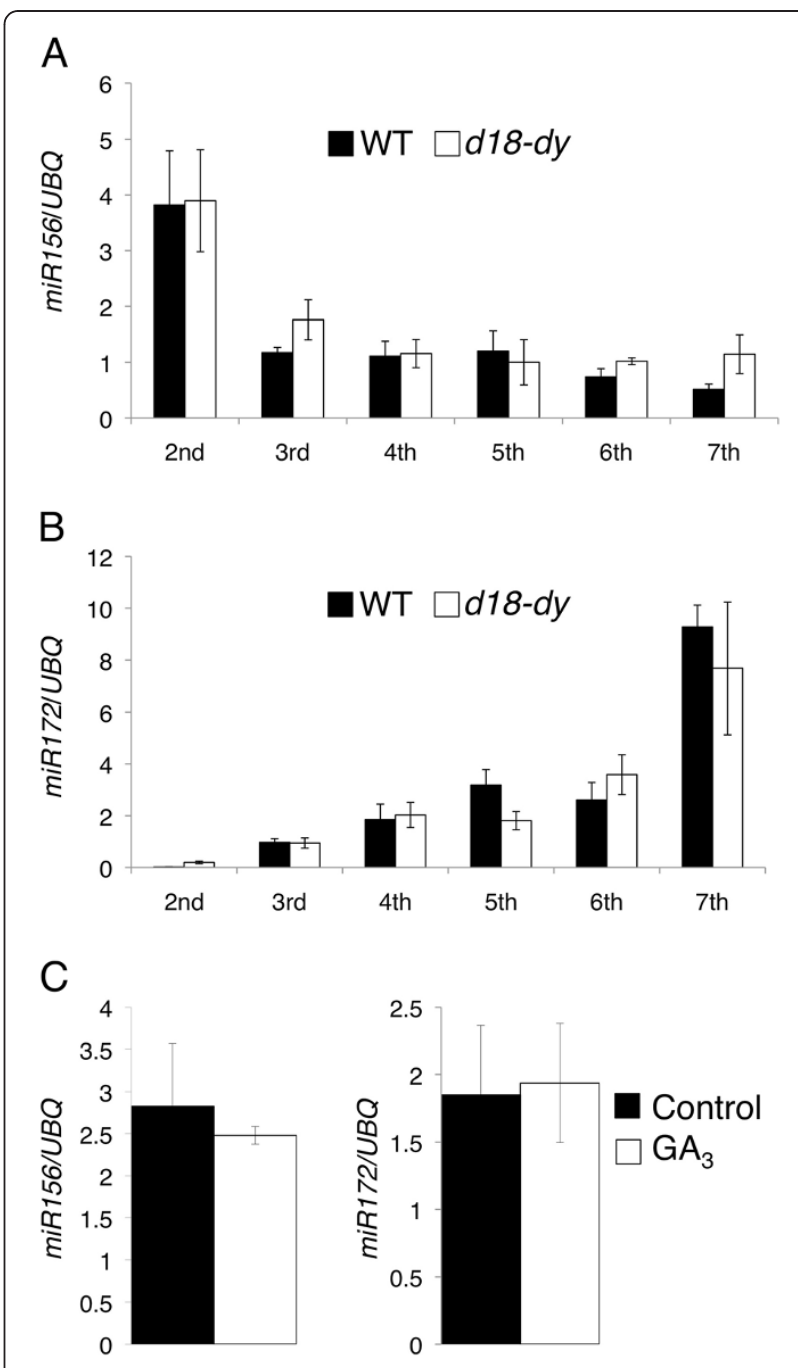

Figure 3 Relationship between two miRNAs and GA. (A)

Expression of miR156 in wild type and d18-dy 2nd to 7th leaves. (B) Expression of miR172 in wild type and d18-dy 2nd to 7th leaves. (C) Expression of miR156 and miR172 in control and $\mathrm{GA}_{3}$ treated 3-dayold plants. Each value is the average of three independent real-time PCR assays. Data in $(\mathbf{A}),(\mathbf{B})$ and $(\mathbf{C})$ represent means $\pm S D(n=3)$.

expression levels of two miRNAs were not affected by the application of $\mathrm{GA}_{3}$ (Figure 3C). Thus I concluded that GA did not regulate the onset of adult phase upstream of miR156.

\section{Expression patterns of OsSPL}

To further confirm the hypothesis that GA functions independently of miR156 pathway in juvenile-adult phase change, I examined the expression levels of OsSPL13 and OsSPL14 that are the ortholog of Arabidopsis SPL3 and SPL9 (Xie et al. 2006). Both OsSPL13 and OsSPL14 contain miR156 target sites, and OsSPL14 ortholog, SPL9 regulates miR172 expression positively in Arabidopsis (Wu et al. 2009). The expression levels of both 
OsSPL13 and OsSPL14 in leaves were almost comparable between wild type and $d 18-d y$ (Figure 4A). Next, I examined OsSPL13 and OsSPL14 expression in $\mathrm{GA}_{3}$ treated plants. Both OsSPL13 and OsSPL14 expression levels were similar between control and $\mathrm{GA}_{3}$ treated plants (Figure 4B). These results indicate that GA does not affect the expression of miR156-target genes. I again confirmed that GA-related pathway did not act upstream of miR156.

\section{Discussion}

\section{GA and miR156 function independently to regulate} juvenile-adult phase change

The study of juvenile-adult phase change is difficult because it is accompanied by subtle morphological traits. In addition, the morphological markers for juvenileadult phase change are different among plant species. However, miR156 and GA are reported as common juvenile-adult phase change regulator in many flowering plants (Lawson and Poethig 1995; Telfer et al. 1997; Wu et al. 2009; Chuck et al. 2007; Wang et al. 2011; Tanaka et al. 2011). GA-related mutant, $d 18-d y$ showed prolonged juvenile phase, such as delayed midrib formation, small leaf size, small SAM size and delayed nodeinternode differentiation. These results strongly indicate that GA also promotes adult phase transition in rice.

The expression patterns of miR156 and miR172 were comparable between wild type and $d 18-d y$ plants. These results suggest that GA and miR156 regulate juvenileadult phase change through independent genetic pathway. Over-expressed MIR156 line in Arabidopsis shows prolonged juvenile phase, however it can enter the reproductive phase (Schwarz et al. 2008). In rice, MIR156 over-expressed plant also showed dwarfism, but developed to flowering stage (Xie et al. 2012). Similarly, GAdeficient mutants can enter the reproductive phase in many flowering plants (Telfer et al. 1997; Itoh et al. 2004). These phenotypes indicate that miR156 functions redundantly with GA in the determination of the exact time of juvenile-adult phase change. Double mutant of MIR156 over-expressed plant and GA deficient mutant might show persistent juvenile phase.

In $d 18-d y$ 6th leaf stage, the plant was obviously dwarf. This indicates that amount of active GA in $d 18-d y$ is still low at 6th leaf stage. However, d18-dy showed normal midrib formation at this stage (Figure 1E). This suggests
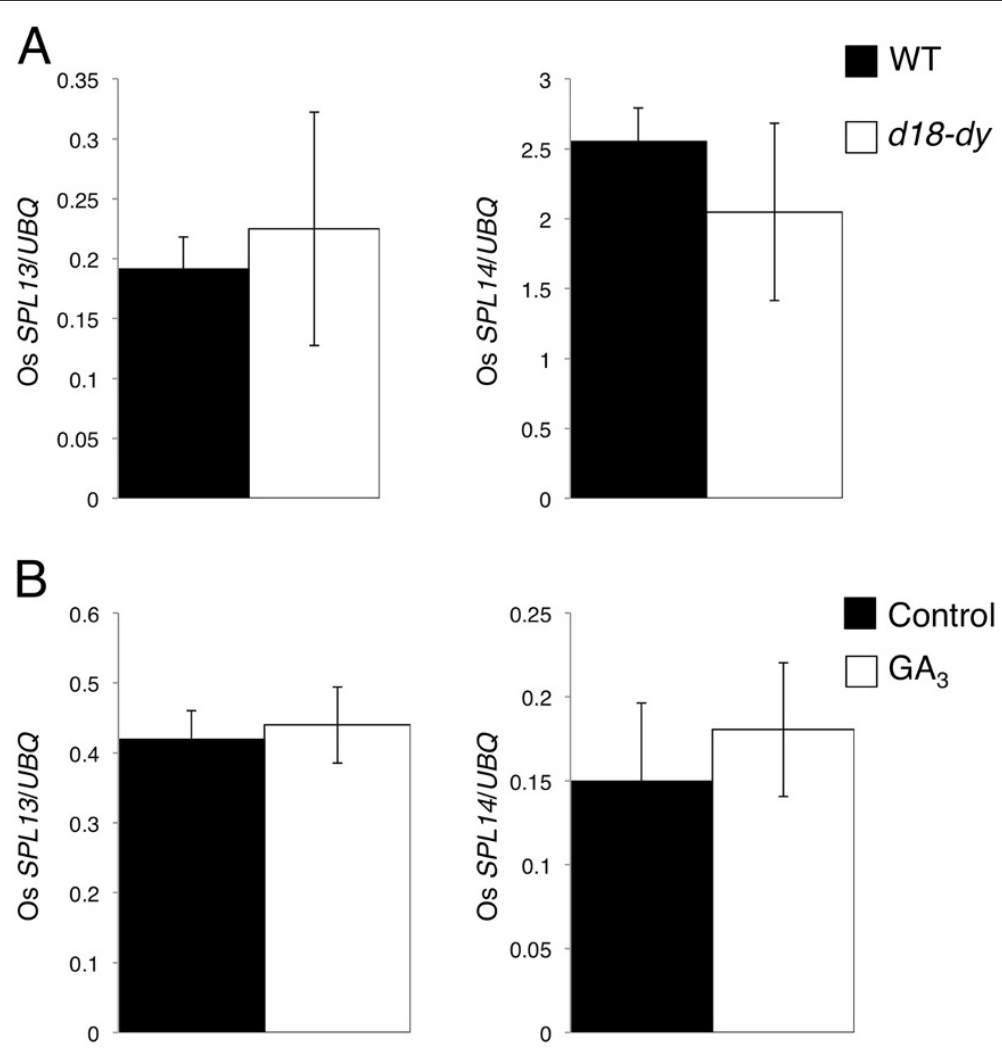

Figure 4 Expression patterns of Os SPLs in $\mathbf{d 1 8 - d y . ~ ( A ) ~ E x p r e s s i o n ~ o f ~ O S ~ S P L 1 3 ~ a n d ~ O s ~ S P L 1 4 ~ i n ~ w i l d ~ t y p e ~ a n d ~ d 1 8 - d y ~ 2 n d ~ l e a v e s . ~ ( B ) ~}$ Expression of Os SPL13 and Os SPL14 in control and GA treated 3-day-old plants. Each value is the average of three independent real-time PCR assays. Data in (A), (B) and $(\mathbf{C})$ represent means \pm SD $(n=3)$. 
that the function of GA is less important for midrib formation after 6th leaf stage than during 2nd-to-5th leaf stages.

Short plastochron is also known as juvenile phase character in rice (Itoh et al. 2005). MIR156 overexpressed plant had more leaves than wild type (Xie et al. 2012). In contrast, the rate of leaf initiation was comparable between $d 18-d y$ and wild type. These indicate that miR156 and GA have different functions in regulation of plastochron.

\section{Conclusions}

Long juvenile phase phenotype of $d 18-d y$ indicated that GA is the adult phase promoter in rice. In higher plants, miR156 and miR172 are also known as juvenile-adult phase change regulator. Our study demonstrated that GA does not regulate juvenile-adult phase change via a pathway of miR156. Moreover, GA does not regulate OsSPLs that are the miR156-target genes.

\section{Methods}

\section{Plant materials}

I used $d 18-d y$ that is a dwarf mutant defective in GA biosynthestic gene encoding GA3 OXIDASE 2. Mutants and wild type plants were grown in pots under natural field conditions.

\section{Paraffin sectioning}

Leaves and shoot apices were fixed with FAA (formalin: acetic acid:50\% ethanol, 1:1:18) for $24 \mathrm{~h}$ at $4^{\circ} \mathrm{C}$. They were dehydrated in a graded ethanol series and embedded in Paraplast plus (McCormick Scientific). Microtome sections ( $8 \mu \mathrm{m}$ thick) were stained with Delafield's hematoxylin.

\section{Gene expression profiling}

The real-time PCR for miR156 and miR172 was performed using TaqMan MicroRNA Assay (Applied Biosystems). Total RNA was extracted using TRIzol reagent (Invitrogen) from wild type 2nd, 3rd, 4th, 5th, 6th and 7th leaves. In addition, RNA was also isolated from $d 18-d y$ 2nd, 3rd, 4th, 5th, 6th and 7th leaves. To quantify the miR156 and miR172 expression, PCR was performed using the TaqMan Fast Universal PCR Master Mix (Applied Biosystem). I used UBQUITIN (UBQ) as inner control and qPCR was conducted using SYBR green master mix (Applied Biosystem). For quantifying the $U B Q$ expression, Real-time PCR was performed using High Capacity RNA-to-cDNA Master Mix (Applied Biosystems).

To observe the Os SPL13 and Os SPL14 expressions, total RNA was isolated from wild type and $d 18-d y$ 2nd leaves. I used $U B Q$ as inner control and qPCR was conducted using SYBR green master mix (Applied Biosystem).
Real-time PCR was performed using High Capacity RNAto-cDNA Master Mix (Applied Biosystems).

For observing miR156, miR172, Os SPLs and GA2ox4. expression patterns in GA treated plants, sterilized seeds of wild type were plated on MS medium (Murashige and Skoog 1962) containing $10^{-5} \mathrm{M} \mathrm{GA}_{3}$ (SIGMA). Plants were grown in a growth chamber under the continuous light at $28^{\circ} \mathrm{C}$. Total RNA was extracted using TRIzol reagent (Invitrogen) from 3-day-old wild type seedlings. To quantify the miR156 and miR172 expression, PCR was performed using the TaqMan Fast Universal PCR Master Mix (Applied Biosystem). I used UBQUITIN $(U B Q)$ as inner control and $\mathrm{qPCR}$ was conducted using SYBR green master mix (Applied Biosystem). For quantifying the Os SPL13, Os SPL14 and UBQ expression, Real-time PCR was performed using High Capacity RNA-to-cDNA Master Mix (Applied Biosystems). PCR was performed using the StepOnePlus real-time PCR system (Applied Biosystems). For quantifying the GA2ox4 and $U B Q$ expression, Real-time PCR was performed using PrimeScript RT Master Mix (Takara). qPCR was conducted using SYBR Premix Ex Taq ll (Takara). PCR was performed using Thermal Cycler Dice TP800 (Takara). Gene-specific primes for Os SPL13, Os SPL14 GA2ox4 and UBQ are listed in Additional file 2: Table S1 online.

\section{Additional files}

Additional file 1: Figure S1. Expression patterns of GA20x4 in GA treated plants. Expression of GA20x4 in control and $\mathrm{GA}_{3}$ treated 3-day-old plants. Each value is the average of three independent real-time PCR assays. Data represent means \pm SD $(n=3)$.

Additional file 2: Table S2. List of primers for semi-quantitative RT-PCR.

\section{Competing interests}

The author declares that they have no competing interests.

\section{Acknowledgement}

I thank Dr. H Kitano (Nagoya Univ.) for kind gift of d18-dy seeds.

Received: 6 July 2012 Accepted: 14 September 2012

Published: 22 September 2012

\section{References}

Asai K, Satoh N, Sasaki H, Satoh H, Nagato Y (2002) A rice heterochronic mutant, mori7, is defective in the juvenile-adult phase change. Development 129:265-273

Chuck G, Cigan AM, Saeteurn K, Hake S (2007) The heterochronic maize mutant Corngrass 1 results from overexpression of a tandem microRNA. Nat Genet 39:544-549

Itoh H, Tatsumi T, Sakamoto T, Otomo K, Toyomasu T, Kitano H, Ashikari M, Ichihara S, Matsuoka M (2004) A rice semi-dwarf gene, Tan-Ginbozu (D35), encodes the gibberellin biosynthesis enzyme, ent-kaurene oxidase. Plant Mol Biol 54:533-547

Itoh J, Nonomura K, Ikeda K, Yamaki S, Inukai Y, Yamagishi H, Kitano H, Nagato Y (2005) Rice plant development: from zygote to spikelet. Plant Cell Physiol 46:23-47 
Lauter N, Kampani A, Carlson S, Goebel M, Moose SP (2005) microRNA172 downregulates glossy15 to promote vegetative phase change in maize. Proc Natl Acad Sci U S A 102:9412-9417

Lawson EJ, Poethig RS (1995) Shoot development in plants: time for a change. Trends Genet 11:263-268

Moose SP, Sisco PH (1996) Glossy15, an APETALA2-like gene from maize that regulates leaf epidermal cell identity. Genes Dev 10:3018-3027

Murashige T, Skoog F (1962) A revised medium for rapid growth and bioassays with tobacco tissue cultures. Physiol Plant 15:473-497

Sakamoto T et al (2004) An overview of gibberellin metabolism enzyme genes and their related mutants in rice. Plant Physiol 134:1642-1653

Sasaki A et al (2002) Green revolution: a mutant gibberellin-synthesis gene in rice. Nature 416:701-702

Schwarz S, Grande AV, Bujdoso N, Saedler H, Huijser P (2008) The microRNA regulated SBP-box genes SPL9 and SPL15 control shoot maturation in Arabidopsis. Plant Mol Biol 67:183-195

Smith MR, Willmann MR, Wu G, Berardini TZ, Moller B, Weijers D, Poethig RS (2009) Cyclophilin 40 is required for microRNA activity in Arabidopsis. Proc Natl Acad Sci U S A 106:5424-5429

Tanaka N, Itoh H, Sentoku N, Kojima M, Sakakibara H, Izawa T, Itoh J, Nagato Y (2011) The COP1 ortholog PPS regulates the juvenile-adult and vegetativereproductive phase changes in rice. Plant Cell 23:2143-2154

Telfer A, Poethig RS (1998) HASTY: a gene that regulates the timing of shoot maturation in Arabidopsis thaliana. Development 125:1889-1898

Telfer A, Bollman KM, Poethig RS (1997) Phase change and the regulation of trichome distribution in Arabidopsis thaliana. Development 124:645-654

Wang JW, Czech B, Weigel D (2009) miR156-regulated SPL transcription factors define an endogenous flowering pathway in Arabidopsis thaliana. Cell 138:738-749

Wang JW, Park MY, Wang LJ, Koo Y, Chen XY, Weigel D, Poethig RS (2011) miRNA control of vegetative phase change in trees. PLoS Genet 7:e1002012

Wu G, Poethig RS (2006) Temporal regulation of shoot development in Arabidopsis thaliana by miR156 and its target SPL3. Development 133:3539-3547

Wu G, Park MY, Conway SR, Wang JW, Weigel D, Poethig RS (2009) The sequential action of miR156 and miR172 regulates developmental timing in Arabidopsis. Cell 138:750-759

Xie K, Wu C, Xiong L (2006) Genomic organization, differential expression, and interaction of SQUAMOSA promoter-binding-like transcription factors and microRNA156 in rice. Plant Physiol 142:280-293

Xie K, Shen J, Hou X, Yao J, Li X, Xiao J, Xiong L (2012) Gradual increase of miR156 regulates temporal expression changes of numerous genes during leaf development in rice. Plant Physiol 158:1382-1394

Yamaguchi S (2008) Gibberellin Metabolism and its Regulation. Annu Rev Plant Biol 59:225-251

doi:10.1186/1939-8433-5-25

Cite this article as: Tanaka: Gibberellin is not a regulator of miR156 in rice juvenile-adult phase change. Rice 2012 5:25.

\section{Submit your manuscript to a SpringerOpen ${ }^{\circ}$ journal and benefit from:}

- Convenient online submission

- Rigorous peer review

- Immediate publication on acceptance

- Open access: articles freely available online

- High visibility within the field

Retaining the copyright to your article

Submit your next manuscript at $\gg$ springeropen.com 